\title{
Sub-1 GHz Flexible Concealed Rectenna Yarn for High-Efficiency Wireless-Powered Electronic Textiles
}

\author{
Mahmoud Wagih, Graduate Student Member, IEEE, Alex S. Weddell, Member, IEEE, \\ and Steve Beeby, Senior Member, IEEE \\ School of Electronics and Computer Science, University of Southampton \\ $\{$ mahm1g15, asw, spb $\} @$ ecs.soton.ac.uk
}

\begin{abstract}
Electronic textiles and seamlessly integrated flexible wearable electronics are an emerging platform for sensing and computing. Batteries and energy harvesters relying on specific materials and transducers are not fully compatible with e-textiles fabrication and large-scale manufacturing. This work proposes a radio frequency energy harvesting rectenna, operating in the sub$1 \mathrm{GHz}$ license-free band, packaged in the form a of rectenna yarn which can be concealed in standard textile weaves. The rectenna yarn is fabricated using thin polyimide copper laminates using photolithography. The rectenna is composed of a $50 \Omega$ meanderline coplanar waveguide monopole antenna and a voltage doubler rectifier, with a lumped matching network. The rectenna achieves 65.8\% RF-DC efficiency and a 8.0-V DC output at 6 and $11 \mathrm{dBm}$ input power, respectively. This is the highest voltage output of a textile wearable rectenna, while maintaining high efficiency down to $-20 \mathrm{dBm}$ and a $-7 \mathrm{dBm} 1-\mathrm{V}$ sensitivity.
\end{abstract}

Index Terms-Antenna, Coplanar Waveguide, Electronic Textile, RF Energy Harvesting, Rectifier, Wireless Power Transfer

\section{INTRODUCTION}

Radio Frequency Energy Harvesting (RFEH) and Wireless Power Transfer (WPT) represent a potential substitute for batteries in low-power Internet of Things (IoT) networks. A textile-based wearable system imposes additional requirements and constraints on the packaging of components such as being flexible, conformable, resilient to bending and crumpling, as well as mechanically durable to withstand daily use [1]. Multiple fabrication methods have been presented for fabricating electronic textiles using additive manufacturing and off-theshelf conductive fabrics [2], [3]. Recently, a production linecompatible technique has been proposed to integrate electronic circuits in textiles in the form of thin, flexible circuityarns which can be incorporated in standard weaves [4] [6]. However, no energy harvesters or Radio Frequency (RF) components have been realised using that technique.

The major bottle-neck in e-textile systems is the power supply unit, where conventional batteries require additional maintenance and are more hazardous compared to passive circuits. While multiple textile-based energy harvesters have been proposed [7], they normally require certain on-body

This work was supported by the European Commission through the project EnABLES: European Infrastructure Powering the Internet of Things, funded under H2020-EU.1.4.1.2. placements for solar cells to receive the highest energy density or for a mechanical harvester to harvest stress or strain.

A RFEH system, composed of conductive traces on a lowcost substrate and a passive rectifier, fulfils the materialrequirements in a textile-based system. Wearable rectennas have been presented using additive manufacturing [8], metallized conductive fabrics [3], and flexible etched Printed Circuit Board (PCB)s [9]. Nevertheless, most reported rectennas were not concealed in the textile for durability, and often rely on certain substrates, such as thick felt fabric to improve the efficiency of patch antennas and a rectifier on a rigid PCB [3].

This work presents, for the first time, a flexible Polyimide antenna filament, with an integrated rectifier, to realise a textile-based RF energy harvester which can be woven into a standard textile yarn. The rectenna is fully compatible with commercial textile fabrication techniques while maintaining high RF-DC Power Conversion Efficiency (PCE) of up to $65.8 \%$ and more than $8 \mathrm{~V} \mathrm{DC}$ output. This paper is structured as follows: section II presents the antenna fabrication technique; the antenna design, simulation and measurements are discussed in section III. The rectifier and rectenna design, simulation, and measurements are in Section IV.

\section{Concealed RF E-TeXtiles FABRication}

The main building block in woven electronic textiles is the conductive e-filament. In this case, the filament is fabricated using an ultra-thin $(25 \mu \mathrm{m})$ Polyimide substrate with a single copper layer. While it is possible to utilize double-sided Kapton or Liquid Crystal Polymer (LCP) copper laminates, the added copper weight significantly affects the flexibility of the yarn, its reliability, and integration in the weave, hence affecting the user's comfort. The typical width of the Polyimide filaments is between 0.1 and $1 \mathrm{~cm}$ to fit seamlessly in bespoke textile pockets, formed during the weaving process [6]. The circuit or antenna filament can alternatively be surrounded by a knitted sheath to form a fully-contained yarn, which can be integrated in conventional textile weaves [5].

Standard photolithography is utilized to etch the traces' pattern onto the filament. A planar substrate, such as a silicon wafer, is generally utilised to keep the flexible substrate rigid 


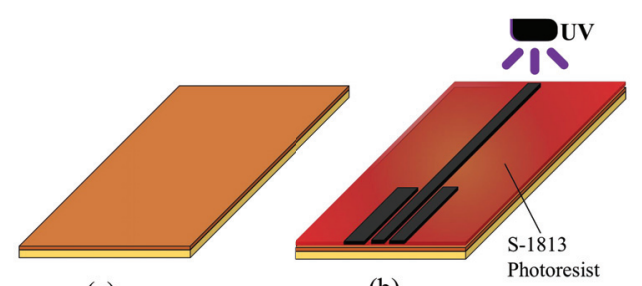

(a)

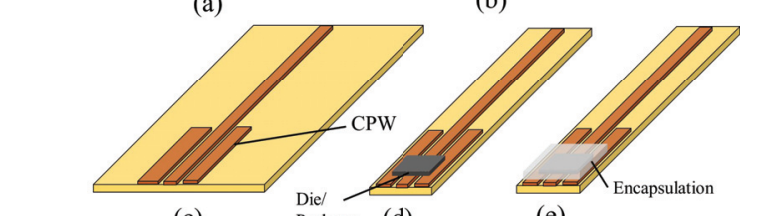

(e)

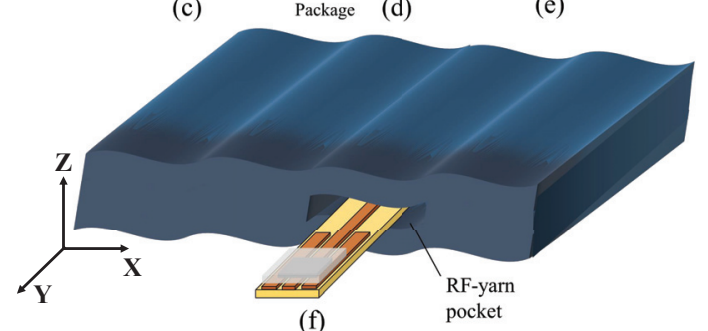

(g)

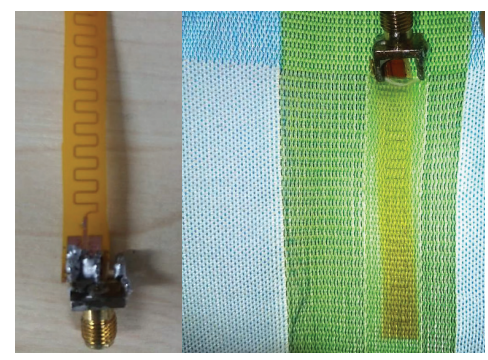

Fig. 1. Fabrication steps of the concealed RF yarn: (a) Commercial polyimide copper laminate, (b): photo-resist coated copper-laminate during UV exposure using a dark-film mask, (c): etched circuit strip, (d): component/die mounting, (e): polymer encapsulation of the discrete components, (f): RF-yarn insertion into a textile-weave pocket, $(\mathrm{g})$ : photograph of the meandered antenna yarn (left), and the concealed antenna under high exposure (right).

during the photo-resist deposition, ultraviolet (UV) exposure, and finally when mounting and encapsulating components. The copper traces are etched in a standard wet-etching acid tank, after developing the photoresist post-UV exposure. Finally, the remaining copper is cleaned using acetone to expose the conductive traces.

The electronic components, either packaged or bare-die, are attached to the tracks using conductive adhesive or solder. The components can then be encapsulated using a variety of polymers for protection from mechanical stress and atmospheric conditions [4]. Fig. 1 shows the steps of the fabrication process of a RF-yarn, in addition to the photograph of the fabricated meander-line Coplanar-Waveguide (CPW) antenna before and after concealment in textile.

While this technique is adequate for DC and low-frequency circuits, when applying it to RF circuits a variety of additional limitations need to be identified and addressed. To begin with, large ground planes are not possible due to the narrow width of the traces. Furthermore, due to the single copper layer structure, the only viable transmission line is a CPW.

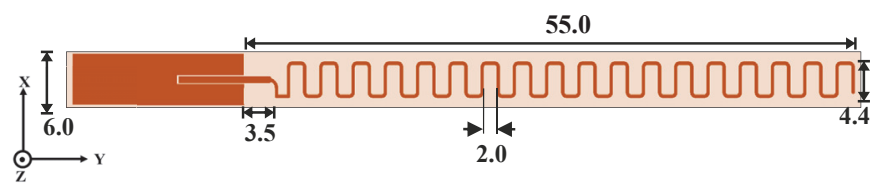

Fig. 2. Layout (not to scale) and dimensions (in $\mathrm{mm}$ ) of the proposed CPW antenna yarn.

Antenna designs in turn, need to be either dipole or monopole antennas with modified geometry to account for the absence or miniaturization of the ground plane.

\section{Antenna Design, Simulation and Measurement}

\section{A. Antenna Design}

As the antenna's geometry is limited in its width, and as a dipole antenna would require a balun to be integrated with a single-ended rectifier, adding to the complexity and weight of the system, the proposed antenna design is a based on a monopole antenna with a compact CPW feed. In order to overcome the absence of a large ground plane, which would degrade the quality of the $50 \Omega$ matching, the quarter-wave monopole has been meandered to increase the antenna's own capacitance. Fig. 2 shows the geometry of the antenna. The antenna has been tuned for a $25 \mu \mathrm{m}$ polyimide substrate with $18 \mu \mathrm{m}$ copper layer and $18 \mu \mathrm{m}$ of epoxy adhesive.

\section{B. Antenna Simulation and Measurement}

The $3 \mathrm{D}$ model of the antenna $(25 \mu \mathrm{m}$ polyimide $+18 \mu \mathrm{m}$ epoxy $+18 \mu$ m copper) has been simulated in CST Microwave Studio to tune the antenna's dimensions and evaluate its farfield performance. The fabricated prototype, excited using an edge mount SMA connector, has been measured using a ZVB4 Vector Network Analyser (VNA). Fig. 3 shows the simulated and measured reflection coefficient, $S_{11}$, of the antenna, showing a $50 \Omega$ matching covering the sub- $1 \mathrm{GHz}$ license-free band $(868 / 915 \mathrm{MHz})$. The computed radiation patterns of the antenna are shown in Fig. 4. The radiation patterns resemble an ideal omnidirectional monopole, making it suitable for receiving and harvesting incident waves without prior knowledge of the transmitter's position. While the high cross-polarization isolation may be decremental when harvesting arbitrarily-polarized waves, two orthogonal antennas can be combined for dual-linear polarization reception.

The main source of discrepancy between the antenna's simulated and measured $S_{11}$ is the SMA's connector size, which is significantly larger than the CPW's ground. In addition, the bending of the antenna (at the connector) and the external foam support required to keep the antenna planar during measurements result in a slight resonance shift, to lower frequencies, due to the higher relative permittivity than vacuum and air. The wider measured bandwidth is attributed to the larger ground, observed by the VNA, due to the SMA connector's body acting as an additional ground.

In order to investigate the effect of integration in the textile weave on the antenna, the antenna has been inserted in the 


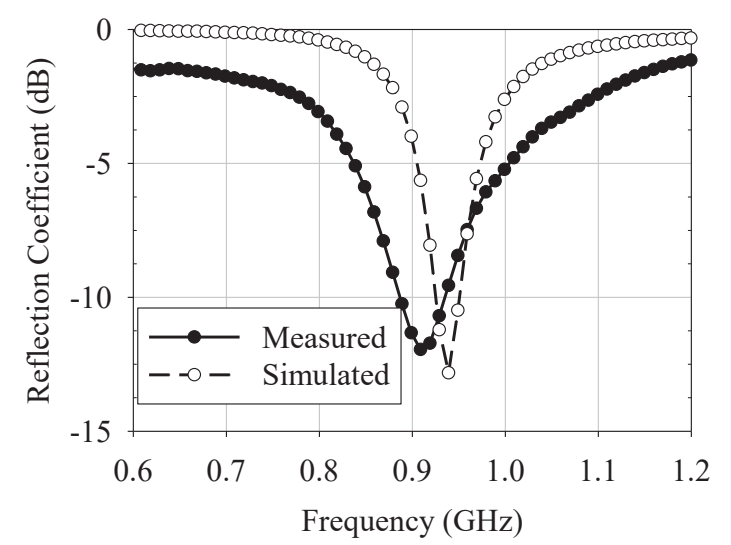

Fig. 3. Simulated and measured reflection coefficient of the CPW antenna yarn in space.
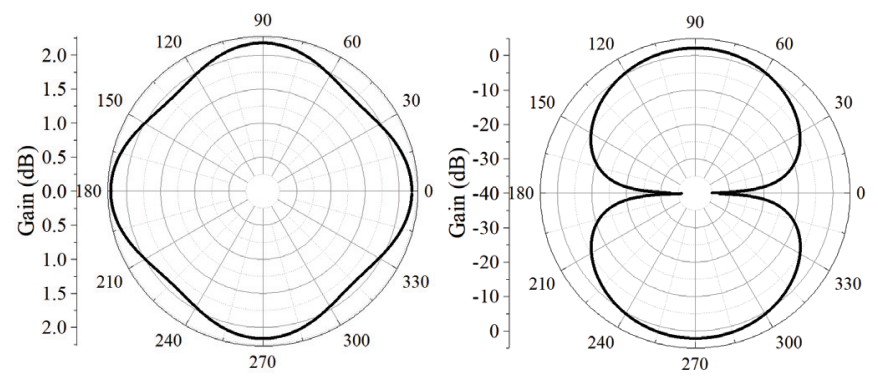

Fig. 4. The CPW antenna yarn gain on the H-plane (left) and the E-Plane (right).

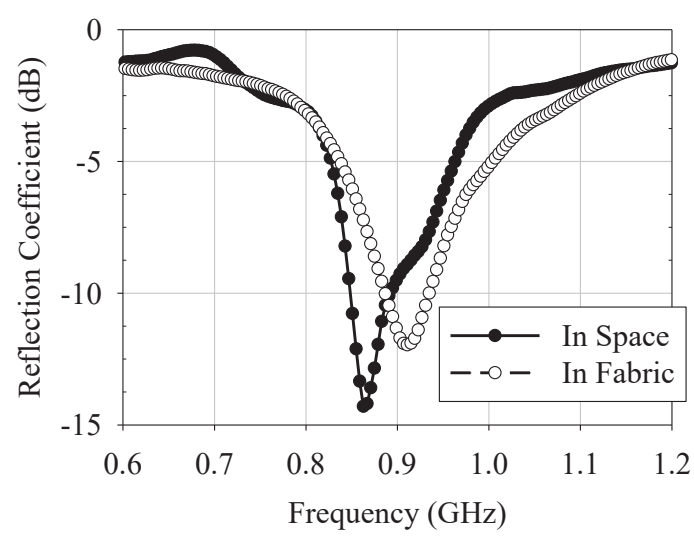

Fig. 5. The measured reflection coefficient of the CPW antenna in space and inside the textile-weave pocket.

bespoke textile pocket, as shown in Fig. 1-g. The $S_{11}$ has been measured with the antenna inside the textile (Fig. 5). The lower reflection observed at frequencies higher than the resonance (915 MHz) can be attributed to the widened bandwidth of the antenna, due to the additional dielectric substrate and superstrate introduced by the textile.

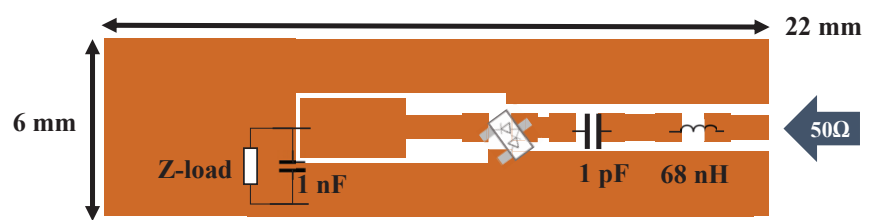

Fig. 6. Layout of the proposed compact CPW flexible rectifier showing the components placement.

\section{Compact CPW Voltage Doubler}

\section{A. Rectifier Design and Matching}

The proposed rectifier is a voltage doubler based on the Infineon BAT15-04r diode. As the package contains two backto-back diodes in a standard SOT-23 package, the realisation of a voltage doubler is simplified while minimising packaging parasitics and the circuit assembly complexity. The trace and gap width of the CPW are identical to the antenna's, discussed in the previous section. A $1 \mathrm{pF}$ RF capacitor, of high selfresonant frequency is used for the voltage doubler's input capacitor.

The rectifier has been designed and simulated using harmonic balance in Keysight ADS. Given the circuit's overall size, distributed L-matching is not possible due to the lack of area to realise components such as open-circuit straight or radial stubs. Moreover, at sub-1 GHz, a distributed elements matching network would have a large length which may increase the losses and reduce the PCE.

Based on the computed large-signal input impedance of the voltage doubler, a single inductor matching network has been designed to match the doubler to $50 \Omega$ at $868 \mathrm{MHz}$. While it is not necessary to match the rectifier's input to $50 \Omega$, as the rectifier's matching could be designed with respect to an antenna of any impedance, a $50 \Omega$ eco-system enables compatibility with standard bench-top test equipment such as signal generators and amplifiers for testing and validation. Fig. 6 shows the layout of the proposed rectifier.

\section{B. Rectifier Simulation and Measurement}

The rectifier's performance has been simulated in ADS as well as measured experimentally, the rectifier has been fed using a $50 \Omega$ source in the simulation as well as in the experiment, and studied under different levels of input RF power, in addition to load resistances. While an ideal series inductor is expected to give an ideal impedance match at the design frequency, as the lumped inductor has a parasitic parallel packaging capacitance (estimated to be $0.14 \mathrm{pF}$ based on the harmonic balance simulation), the minimum achievable reflection coefficient is around $-10 \mathrm{~dB}$ at $810 \mathrm{MHz}$.

In order to evaluate the performance of the rectifier, a $50 \Omega$ RF power source: a VNA in Continuous Wave $(\mathrm{CW})$ generator mode, has been used to test the rectifier's performance at different power levels while observing the DC output. The advantage of using a $50 \Omega$ power source compared to testing an integrated rectenna is that $\mathrm{CW}$ generators can be calibrated to have a maximum error of $\pm 0.1 \mathrm{~dB}$ as opposed to around \pm 1 

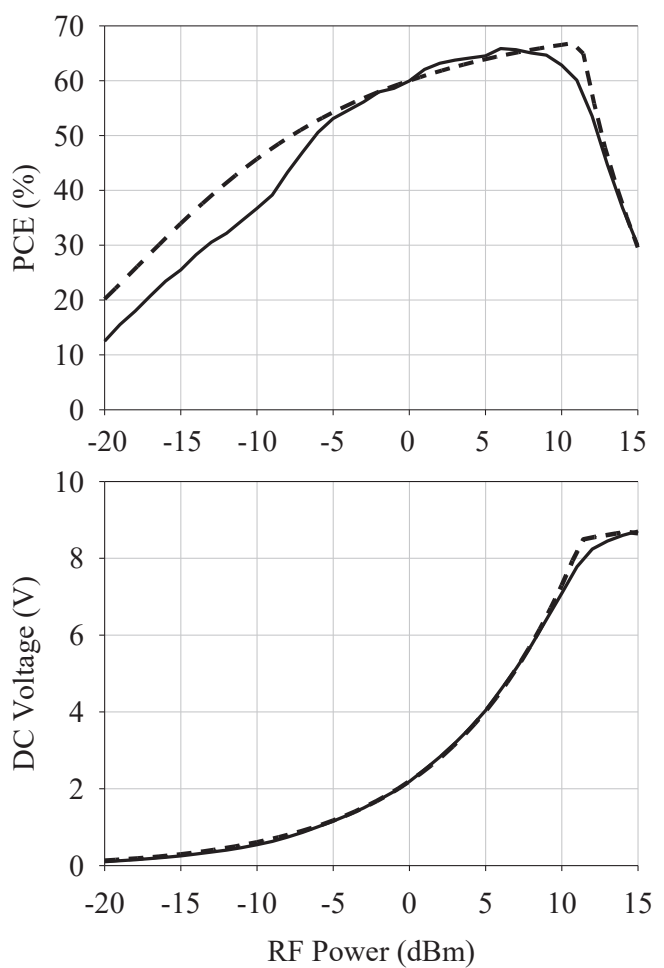

Fig. 7. Simulated (dashed) and measured (solid) DC output of the CPW rectifier yarn: RF-DC efficiency (top) and voltage across $8 \mathrm{k} \Omega$ load (bottom)

$\mathrm{dB}$ when using an incident plane-wave or a reference antenna to estimate the received power [3]. Fig. 7 shows the PCE and DC output voltage of the rectifier, with a peak PCE of $65.8 \%$ and more than $8-\mathrm{V}$, at 6 and $11 \mathrm{dBm}$ respectively. The proposed rectifier has a $-7 \mathrm{dBm} 1-\mathrm{V}$ sensitivity demonstrating its suitability for sub-mW RFEH applications.

The final step in validating the performance of the rectenna is calculating the bandwidth of the rectifier referenced to the antenna's impedance $\left(Z_{\text {ant }}\right)$. To illustrate as the antenna's impedance at $868 \mathrm{MHz}$ is $Z_{\text {ant }}=42.4-j 41.2 \Omega$, the bandwidth of the rectifier needs to be calculated at that source impedance and not the VNA's or CW generator's $50 \Omega$ impedance. Fig. 8 shows the calculated bandwidth of the rectifier, obtained from exciting the large-signal s-parameters object of the rectifier (obtained as a touchstone file) using a power source of $Z_{0}=Z_{\text {ant }}$. Given the antenna's simulated radiation efficiency $\left(\eta_{\text {Rad. }}\right)$ of $96 \%$, the combined antennarectifier PCE can be calculated using (1).

$$
P C E_{\text {Rectenna }}=\eta_{\text {Rad. }} \times \frac{P_{D C}}{P_{R F}}
$$

The calculated total rectenna efficiency (1) is $63.2 \%$ at 6 $\mathrm{dBm}$ input power. Table I shows a comparison of the proposed rectenna with state-of-the-art wearable rectennas.

While reported rectennas achieve similar efficiencies by using a single series rectifier based on a diode of lower turn-on and forward voltage (SMS7630), the output voltage in [10] and [11] will be limited to less than $2-\mathrm{V}$, due to the low breakdown

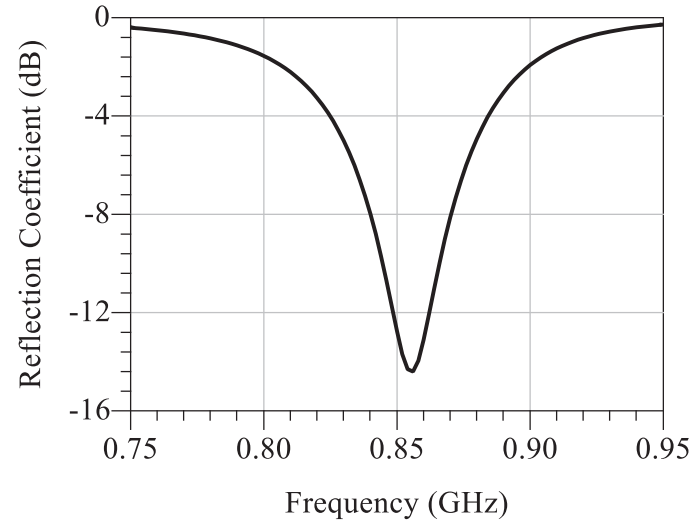

Fig. 8. Rectenna's bandwidth: the rectifier's bandwidth referenced to a source of impedance $Z_{0}=Z_{\text {ant }}$.

TABLE I

COMPARISON OF THE PROPOSED RECTENNA WITH STATE-OF-THE-ART FLEXIBLE AND TEXTILE RECTENNAS

\begin{tabular}{llll}
\hline & This work & $\begin{array}{l}\text { TMTT 2018 } \\
{[12]}\end{array}$ & IMS 2019 [11] \\
\hline Freq $(\mathrm{GHz})$ & 0.868 & 0.9 & 2.4 \\
\hline Fabrication & $\begin{array}{l}\text { Flexible textile } \\
\text { yarn }\end{array}$ & $\begin{array}{l}\text { Copper tape on } \\
\text { paper }\end{array}$ & $\begin{array}{l}\text { Conductive } \\
\text { fabric }\end{array}$ \\
\hline Antenna design & CPW yarn & Coupled slot & Patch \\
\hline Dimensions & $0.22 \lambda_{0} \times$ & $0.33 \lambda_{0} \times$ & $0.56 \lambda_{0} \times$ \\
(relative to $\left.\lambda_{0}\right)$ & $0.17 \lambda_{0} \times$ & $0.33 \lambda_{0} \times$ & $0.54 \lambda_{0}$ \\
& $0.001 \lambda_{0}$ & $0.001 \lambda_{0}$ & \\
\hline Max PCE at & $65.8 \%$ at 6 & $\begin{array}{l}57 \% \text { at }-1.3 \\
\text { dBm }\end{array}$ & $70 \%$ at $8 \mathrm{dBm}$ \\
$\mathrm{P}_{R F}$ & $\mathrm{dBm}$ & $1.6 \mathrm{~V}$ at $0 \mathrm{dBm}$ & $\mathrm{NR}$ \\
\hline Max DC V at & $8 \mathrm{~V}$ at $11 \mathrm{dBm}$ & & \\
$P_{R F}$ & & &
\end{tabular}

voltage of the diode. The proposed voltage doubler rectenna is capable of both achieving high efficiency (more than 60\%) at $5 \mathrm{dBm}$, as well as generating an output voltage of more than $8-\mathrm{V}$ above $10 \mathrm{dBm}$ with a sub-mW $1-\mathrm{V}$ sensitivity.

\section{CONCLUSION}

This paper has presented a new technique to fabricate concealed antennas and RF circuits, demonstrating a CPW rectenna yarn as a power supply unit for reliable e-textiles. The proposed antenna filament meets the packaging requirements of e-yarns and maintains its bandwidth inside and outside the textile weave. A miniaturized CPW voltage doubler is presented for integration with the proposed antenna or with a standard $50 \Omega$ source, demonstrating the first fully-concealed antenna and RFEH-yarn, and achieving the highest DC voltage and efficiency while occupying minimal area and maintaining an ultra-low profile.

\section{REFERENCES}

[1] K. Yang, R. Torah, Y. Wei, S. Beeby, and J. Tudor, "Waterproof and durable screen printed silver conductive tracks on textiles," Textile Research Journal, vol. 83, pp. 2023 - 2031, 2013.

[2] W. G. Whittow, A. Chauraya, J. C. Vardaxoglou, Y. Li, R. Torah, K. Yang, S. Beeby, and J. Tudor, "Inkjet-printed microstrip patch antennas realized on textile for wearable applications," IEEE Antennas Wireless Propag. Lett., vol. 13, pp. 71-74, 2014. 
[3] S.-E. Adami, P. Proynov, G. S. Hilton, G. Yang, C. Zhang, D. Zhu, Y. Li, S. P. Beeby, I. J. Craddock, and B. H. Stark, "A Flexible 2.45GHz Power Harvesting Wristband With Net System Output From -24.3 dBm of RF Power," IEEE Trans. Microw. Theory Techn., 2018.

[4] M. Li, J. Tudor, J. Liu, R. Torah, A. Komolafe, and S. Beeby, "Novel electronic packaging method for functional electronic textiles," IEEE Transactions on Components, Packaging and Manufacturing Technology, vol. 9, no. 2, pp. $216-225,2019$.

[5] D. A. Hardy, I. Anastasopoulos, M.-N. Nashed, C. Oliveira, T. HughesRiley, A. Komolafe, J. Tudor, R. Torah, S. Beeby, and T. Dias, "Automated insertion of package dies onto wire and into a textile yarn sheath," Microsystem Technologies, pp. 1 - 13, 2019.

[6] A. Komolafe, Y. Torah, Russel ans Wei, H. Nunesmatos, M. Li, D. Hardy, T. Dias, M. Tudor, and S. Beeby, "Integrating flexible filament circuits for etextile applications," Advanced Materials Technologies, vol. 4, no. 7, 2019

[7] S. Lemey, F. Declercq, and H. Rogier, "Textile antennas as hybrid energy-harvesting platforms," Proceedings of the IEEE, vol. 102, no. 11, pp. 1833 - 1857, 2014.

[8] S.-E. Adami, D. Zhu, Y. Li, E. Mellios, B. H. Stark, and S. Beeby, "A $2.45 \mathrm{GHz}$ rectenna screen-printed on polycotton for on-body RF power transfer and harvesting," in 2015 IEEE Wireless Power Transfer Conference (WPTC), 2015

[9] J. Bito, J. G. Hester, and M. M. Tentzeris, "Ambient rf energy harvesting from a two-way talk radio for flexible wearable wireless sensor devices utilizing inkjet printing technologies," IEEE Trans. Microw. Theory Techn., vol. 63, 12, pp. 4533-4543, 2015.

[10] V. Palazzi, M. D. Prete, and M. Fantuzzi, "Scavenging for Energy: A Rectenna Design for Wireless Energy Harvesting in UHF Mobile Telephony Bands," IEEE Microwave Magazine, vol. 18, 1, pp. 91 - 99, 2017.

[11] D. Vital, S. Bhardwaj, and J. L. Volakis, "A $2.45 \mathrm{GHz}$ RF Power Harvesting System Using Textile-Based Single-Diode Rectennas," in 2019 IEEE MTT-S International Microwave Symposium (IMS), 2019.

[12] V. Palazzi, J. Hester, J. Bito, F. Alimenti, C. Kalialakis, A. Collado, P. Mezzanotte, A. Georgiadis, L. Roselli, and M. M. Tentzeris, "A Novel Ultra-Lightweight Multiband Rectenna on Paper for RF Energy Harvesting in the Next Generation LTE Bands," IEEE Trans. Microw. Theory Techn., vol. 66, 1, pp. $366-379,2018$ 